\title{
Comment on: A British Ophthalmic Surveillance Unit (BOSU) study into dysthyroid optic neuropathy in the United Kingdom
}

\author{
Jonathan C. P. Roos ${ }^{1,2,3} \cdot$ Rachna Murthy ${ }^{1,2,3}$
}

Received: 26 November 2018 / Accepted: 26 November 2018 / Published online: 12 December 2018

(c) The Royal College of Ophthalmologists 2018

In their British Ophthalmic Surveillance Unit (BOSU) study on dysthyroid optic neuropathy (DON), Wong and colleagues [1] report an annual incidence of 0.75 per million per annum, i.e. around 50 patients per year in the UK, which fits with previously published data [2]. Surprisingly however, almost half of their patients who were followed up for 9 months went on to have orbital decompression surgery (47\%), despite initial medical therapy—and with a mean delay of only 2.5 months post presentation [1].

We should like to contrast this need for swift surgical intervention with evidence from our centre where we have for long used an alternative, ciclosporin-based medical regimen, unlike the BOSU study which reported predominantly i.v. methylprednisolone followed by oral steroid and occasional addition of radiotherapy (two patients), rituximab (1) or azathioprine (1). We retrospectively reviewed electronic case records spanning 23 years from our unit and identified 522 patients with thyroid eye disease (TED), of which 145 were considered sight-threatening as defined by EUGOGO [3], and 75 had DON. During the 12month period of the BOSU study we saw one patient with DON at presentation. All 75 DON patients were followed up for between 2 and 10 years and had been treated with the Cambridge regimen: three pulses of i.v. methylprednisolone $10 \mathrm{mg} / \mathrm{kg}$ at 48 -hour intervals, followed by low dose tapering oral prednisolone, starting at $30 \mathrm{mg}$, then 25,20 , $17.5,15 \mathrm{mg}$ (3 weeks at each dose) then subsequent reduction by $1 \mathrm{mg}$ per month, and ciclosporin A (Neoral) starting at $2 \mathrm{mg} / \mathrm{kg}$ twice daily. As part of the regimen we

Rachna Murthy

rachna.murthy@addenbrookes.nhs.uk

1 Thyroid Eye Disease Service, Department of Ophthalmology, Cambridge University Hospitals NHS Foundation Trust,

Cambridge, UK

2 Department of Ophthalmology, Ipswich Hospital, Heath Road, Ipswich, UK

3 Department of Clinical Neurosciences, University of Cambridge, Cambridge, UK aim for a ciclosporin trough level of 150-200 micrograms/L during the first six months, then 100 to $150 \mathrm{mcg} / \mathrm{L}$ for the second six months and $50-100 \mathrm{mcg} / \mathrm{L}$ thereafter. Ciclosporin is then gradually withdrawn over six months after prednisolone the has been stopped.

Seventy of the 75 DON patients (93\%) recovered their premorbid visual acuities and 69 of 75 patients (92\%) recovered premorbid visual fields. Interestingly, only five patients required surgical decompression of their orbits following our medical regime, with all five having presented with complicated or delayed presentations.

These data point to a potential role for ciclosporin in helping to reduce the need for orbital decompression surgery in DON. Previously, we have shown that ciclosporin therapy rapidly reduces the circulating anti-TSH receptor antibody, further evidence of its utility for TED [4]. Orbital wall decompression is associated with significant risks: the BOSU study reports one patient who developed perception of light vision following surgery and another developed intractable diplopia.

Acknowledgements The authors would like to acknowledge helpful discussions with Drs. Nima Ghadiri and Paul Meyer.

\section{Compliance with ethical standards}

Conflict of interest The authors declare that they have no conflict of interest.

\section{References}

1. Wong Y, Dickinson J, Perros P, Dayan C, Veeramani P, Morris D, et al. A British Ophthalmological Surveillance Unit (BOSU) study into dysthyroid optic neuropathy in the United Kingdom. Eye. 2018;32:1555-62.

2. Perros P, Hegedius L, Bartalena L, Marocci C, Kahaly GJ, Baldeschi L, et al. Graves' Orbitopathy as a rare disease in Europe: a European Group on Graves' Orbitopathy (EUGOGO) position statement. Orphanet J Rare Dis. 2017;12:72.

3. Bartalena L, Baldeschi L, Dickinson A, Eckstein A, Kendall-Taylor P, Marcocci C, et al. European Group on Graves' Orbitopathy 
(EUGOGO). Consensus statement of the European Group on Graves' orbitopathy (EUGOGO) on management of GO. Eur J Endocrinol. 2008;158:273-85.

4. Roos JCP, Paulpandian V, Murthy R. Serial TSH-receptor antibody levels to guide the management of thyroid eye disease: the impact of smoking, immunosuppression, radio-iodine, and thyroidectomy. Eye (Lond). 2018 Nov 6. doi: 10.1038/s41433-018-0242-9. [Epub ahead of print]

\title{
Eye and appearance characteristics of encephalocraniocutaneous lipomatosis
}

\author{
${\text { Yanming } \operatorname{Tian}^{1} \cdot \text { Yi Wang }^{2} \cdot \text { Xiaowei Gao }}^{1} \cdot$ Yulong Zhang ${ }^{1} \cdot$ Yan Ju $^{1}$
}

Received: 8 August 2018 / Accepted: 15 August 2018 / Published online: 24 September 2018

(c) The Royal College of Ophthalmologists 2018

Encephalocraniocutaneous lipomatosis (ECCL) is a rare neurocutaneous syndrome. The unique clinical manifestations of the disease include ocular edema or hamartoma, abnormal skin on the head and neck, and corresponding hair loss on the scalp. Historical literature on ECCL consists mainly of case reports and does not present the common features of the disease. We present the characteristic manifestations of the eye and skin to provide ophthalmologists with a fuller understanding of in ECCL.

\section{Clinical information}

Four patients, all male, aged 3-18 years, presented with ophthalmologic disorders caused by local or diffuse hyperplasia of the ocular surface and had irregular granule-like lesions on the head or neck and unilateral visible granular raised lesions on the tongue mucosal surface (Fig. 1). No brain abnormality was apparent on

Yanming Tian

tianyanming@163.com

$\triangle$ Xiaowei Gao

Gaoxiaowei@163.com

$\triangle$ Yulong Zhang

zhangyl112@163.com

$\triangle$ Yan Ju

juyan474@163.com

1 No. 474 Hospital of the Chinese People's Liberation Army (PLA), Urumqi 830012, China

2 Orbital Disease Institute, Armed Police General Hospital, Beijing 100039, China skull computed tomography, and focal calcification was visible on the wall of the eye. All patients had normal intelligence and no history of seizures. ocular surface tumor was removed with conjunctival sac reconstruction or lamellar keratoplasty.

Pathological results: Two cases were corneal Sebaceous tumor (mature squamous tumor) and two cases were fibrous epithelial polyps.

\section{Discussion}

ECCL is a rare neurocutaneous syndrome with unknown etiology and multiple sporadic differences in ethnicity, gender, and family history. Since Haberland [1] described this syndrome in 1970, more than 60 instances of related disorders have been published worldwide, mainly reported via pediatrics, and neurosurgery cases [2, 3]. The main features of the syndrome include asymmetric skin damage to the head and neck, central nervous system abnormalities(CNS), ocular abnormalities, often bilaterally. hair loss in the local skin lesion area. The most common eye lesions in ECCL are ocular tumors around the eyeball and small skin nodules. Other rare eye abnormalities of the syndrome such as bilateral optic disk defects and lack of bilateral iris eyelid retraction have been reported [4].

The occurrence of ECCL may be related to genetic variation, and it is speculated that autosomal gene mutations lead to ectodermal dysplasia, while chimerism may be the cause of mesenchymal tumors and angiogenesis. 
Fig. 1 Appearance of ECCL. A: Case 1. Left eye

subconjunctival multifocal pink raised lesions. B: Case 2. Right corneal surface and left subconjunctival pink focal, smooth, bulging lesion. C: Case 3. Pink corneal smooth bulging lesion on the left cornea.

D: Case 4. right eye conjunctiva, and substantial freshly appearing protrusion on the right side of the skin. E,F: case 1 and case 2 . Side view: left scalp, face, and neck skin lesions and hairless area. G: case 4. Black lesions on the ipsilateral neck. H: Granular bulge of the left tongue mucosa
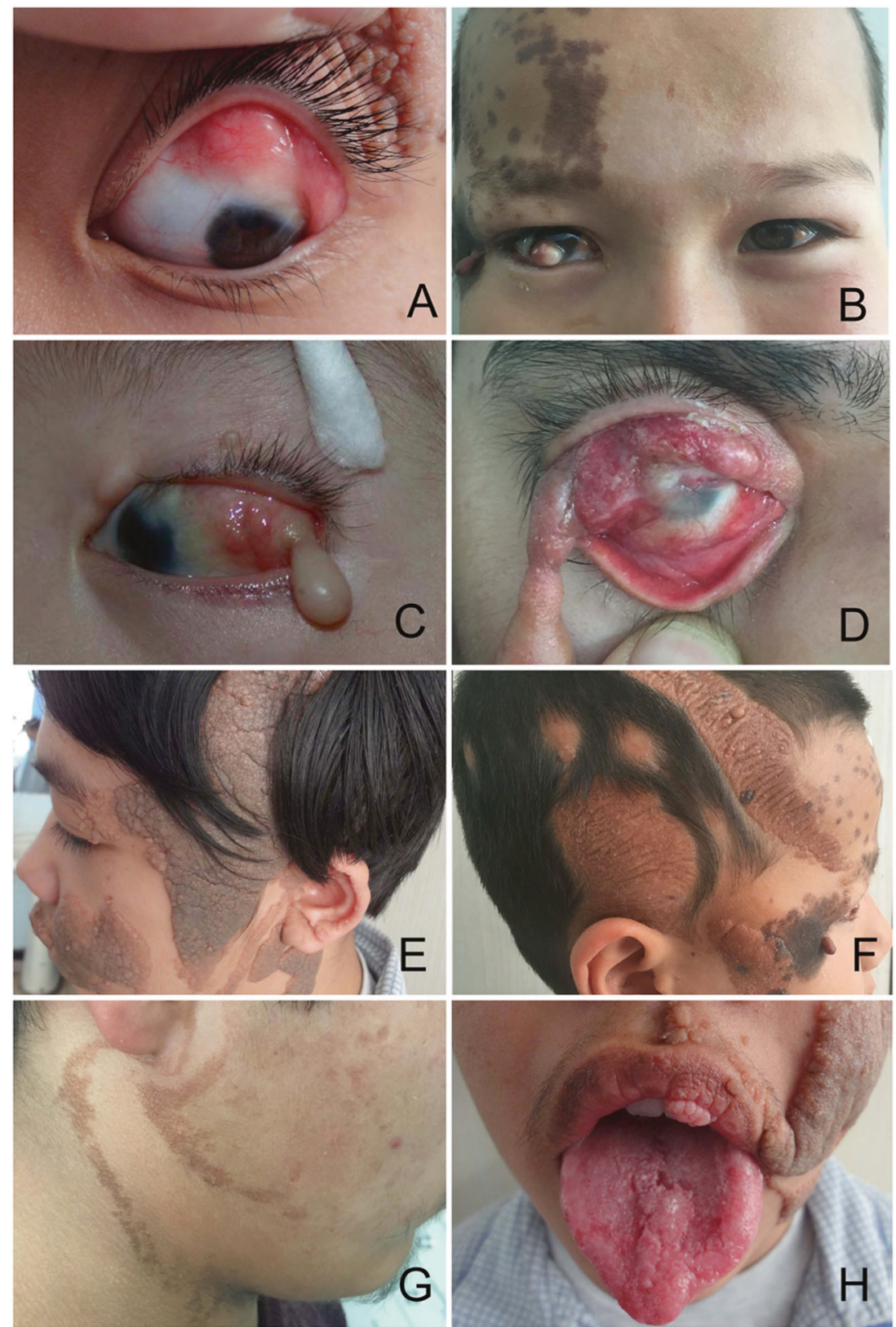

Syndromes that need to be differentiated from linear nevus sebaceous syndrome (LNS), and oculocerebrocutaneous syndrome (OCC). A diagnosis of OCC needs to include CNS cysts or hydrocephalus, small eyeballs with orbital cysts. Skin lesions of LNS are mainly located in the midline of the face forming a linear pattern. According to the revised ECCL diagnostic criteria (Table 1) by Moog [5] in 2009, The ECCL diagnosis of these four cases is clear.

This group of patients shared the following common clinical features of ECCL: (1) ocular lesions; (2) flaky granular pigmentation of the area of skin loss; (3) granular mucosa visible on the surface of the tongue not observed in the previous literature. Ophthalmologists should consider the diagnosis of ECCL in their assessment if they encounter patients with these common characteristics.

There is no specific treatment for ECCL. The main ophthalmic approach is surgical removal of local bud tumors, with improvement of the cosmetic appearance as far as possible. 


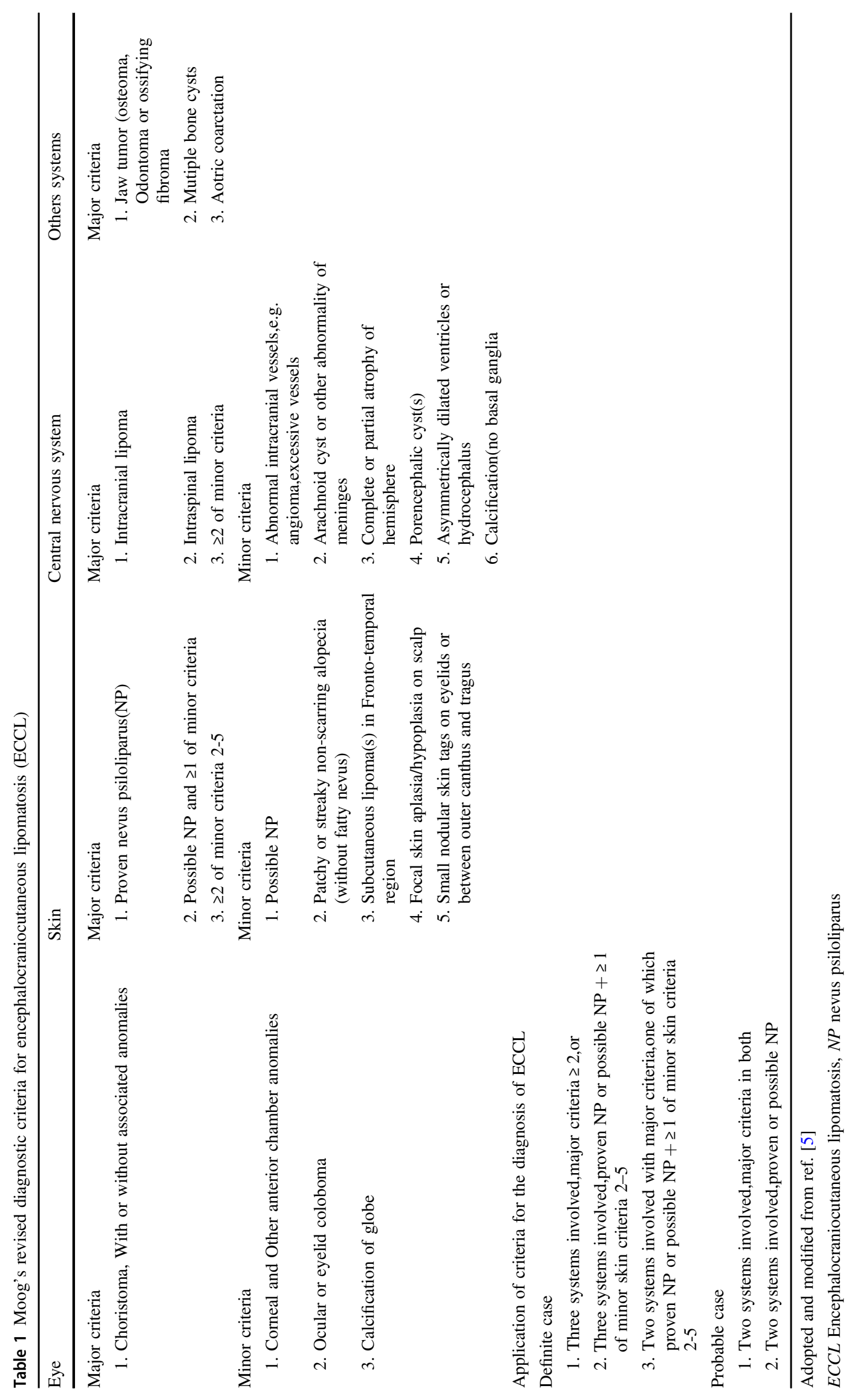




\section{Compliance with ethical standards}

Conflict of interest The authors declare that they have no conflict of interest.

\section{References}

1. Haberland C, Perou M. Encephalocraniocutaneous lipomatosis. A new example of ectomesodermal dysgenesis. Arch Neurol. 1970;22:144-55.
2. Bavle A, Shah R, Gross N, et al. Encephalocraniocutaneous Lipomatosis. J Pediatr Hematol Oncol. 2018. Epub ahead of print.

3. Muñoz-Montufar JP, Rayo-Mares JD, Reyes-Cuayahuitl A. et al. Lipomatosis encefalocraneocutánea: reporte de caso. Gac Med Mex. 2017;153:915-8.

4. Almer Zina, Vishnevskia-Dai Victoria, Zadok David. et al. Encephalocraniocutaneous lipomatosis: case report and review of the literature. Cornea. 2003;22:389-390.

5. Moog U. Encephalocraniocutaneous lipomatosis. J Med Genet. 2009;46:721-9.

\title{
Comment on: Proposal for a new classification for ocular surface squamous neoplasia
}

\author{
Lindsay A. McGrath ${ }^{1}$. Sachin M. Salvi $\mathbb{B}^{1}$ \\ Received: 4 September 2018 / Accepted: 4 October 2018 / Published online: 18 October 2018 \\ (c) The Royal College of Ophthalmologists 2018
}

We read with interest Meel and Dhiman's recent proposal for a new classification for ocular surface squamous neoplasia (OSSN) [1]. The authors offer a modified criteria for conjunctival neoplasia that takes into account invasion (on ultrasound biomicrosopy (UBM)) and provides a rough guide for treatment. They suggest that OSSN of Grade III (with intraocular involvement present) should be managed with enucleation.

The potential of advanced ocular surface squamous lesions to invade through the sclera or cornea is well recognised with enucleation being the traditional management [2]. However, many studies over the past decade have shown success with globe-sparing therapy for these advanced lesions-and it is certainly our experience that invasive squamous lesions can be managed in this way [3]. Shields et al. showed that plaque brachytherapy is a safe and reliable alternative to globe removal for eyes with conjunctival squamous cell carcinoma (SCC) showing scleral invasion and/or intraocular involvement [2]. Further, Graue et al. demonstrated local tumour control in $75 \%$ of

Lindsay A. McGrath

lindsay.mcg@gmail.com

1 Sheffield Ocular Oncology Service, Royal Hallamshire Hospital, Sheffield S10 2JF, UK recalcitrant conjunctival SCCs treated with electron beam radiotherapy [4].

The relevance of the American Joint Committee on Cancer (AJCC) staging of conjunctival SCC in guiding initial management was challenged in a recent work by Belleverie et al. [5]. They also suggest reclassification of the T3 category (diffuse vs deep invading) to better guide initial treatment. However, similar to previous reports, these authors also advocate the use of surface brachytherapy for scleral invasion of SCC [5].

The AJCC publications are recognised as authoritative guides for cancer staging and communicating information about cancer. Evidence-based staging is important to guide therapy and define prognosis, and the AJCC aims to help doctors design a treatment plan for individual patients. Meel and Dhiman's proposal include treatment in their modified staging system, and, although these management options are perhaps not available in their centre, we feel it would be useful to include staged options of therapy for advanced lesions as alternative to enucleation [1]. There appears to be an error in the classification table, listing Grade II lesions incorrectly as having no invasion into ocular coats on imaging, which contradicts the imaging findings and manuscript definitions of this grade.

We support the current classification system published by the AJCC, but agree with recent studies suggesting reclassification of the T3 category [5]. 


\section{Compliance with ethical standards}

Conflict of interest The authors declare that they have no conflict of interest.

\section{References}

1. Meel R, Dhiman R. Proposal for a new classification for ocular surface squamous neoplasia. Eye. 2018;32:1284-5.

2. Shields CL, Chien JL, Surakiatchanukul T, Sioufi K, Lally SE, Shields JA. Conjunctival tTumors: review of clinica features, risks, biomarkers, and outcomes - the 2017 J.Donald M. Gass LLecture. Asia Pac J Ophthalmol. 2017;6:109-20.

3. El-Assal KS, Salvi SM, Rundle PA, Mudhar HS, Rennie IG. Treatment of invasive ocular surface squamous neoplasia with proton beam therapy. Eye. 2013 Oct;27(10):1223.

4. Graue GF, Tena LB, Finger PT. elElectron beam radiation for conjunctival squamous carcinoma. Ophthal Plas Reconstr Surg. 2011;27:277-81.

5. Bellerive C, Berry JL, Polski A, Singh AD. Conjunctival squamous neoplasia: staging and initial treatment. Cornea. 2018. https://doi. org/10.1097/ICO.0000000000001651.

\title{
Response to 'Comment on: Proposal for a new classification for ocular surface squamous neoplasia'
}

\author{
Rachna Meel ${ }^{1} \cdot$ Rebika Dhiman $^{1}$ \\ Published online: 19 October 2018 \\ (c) The Royal College of Ophthalmologists 2018
}

We thank McGrath and Salvi [1] for their valuable comments and suggestions.

There are no published guidelines for treatment of ocular surface squamous neoplasia (OSSN). Also, clinical spectrum of OSSN varies significantly between different continents like Africa and North America or Australia [2]. Clinical presentation is significantly delayed in resource-constrained countries, and hence use of conservative management options like plaque brachytherapy are not only limited by availability but also by feasibility.

The conventional method of treatment for OSSN is wide local surgical excision using a 'no touch technique' and cryotherapy of conjunctival edges. Topical treatment with chemotherapeutic agents and interferons has become popular in the past 1-2 decades due to the inherent benefits of topical treatment-treatment of a complete ocular surface, thus reducing the chances of microscopic residual disease, lesser side effects of treatment as compared to surgical excision, especially in giant or diffuse tumors and the relative ease of

Rachna Meel

dr.rachnameel@gmail.com

1 Oculoplasty and Ocular Oncology Services, Dr R P Centre for Ophthalmic Sciences, All India Institute of Medical Sciences, New Delhi 110029, India administering treatment. Traditionally, enucleation is done for tumors with intraocular extension and exenteration for OSSN with orbital extension.

We agree with the authors that, technology-dependent treatment modalities, like plaque brachytherapy and proton beam therapy may not be available in resource-constrained countries. There are adequate reports in literature to show that brachytherapy effectively controls OSSN invading into the ocular coats and hence may be used as an adjuvant treatment in cases with incomplete tumor resection (Table 1) (the same has been added in the treatment options for grade II tumor in the revised table) [3]. However, its use in OSSN with overt (obvious on imaging (UBM) or clinically) intraocular extension is still being explored and needs to be evaluated in further studies [4]. We, at our center are now using adjuvant plaque brachytherapy in cases of invasive SCC, where there is microscopic residual disease in the ocular coats after surgery.

The proposed classification attempts to address the concerns with AJCC classification, as reported by previous studies and intends to give a rough guideline for treatment based on most commonly available treatment modalities [4]. While enucleation and exenteration remain the most common method of treatment for tumor with ocular and orbital extension, respectively, the treatment scenario for OSSN with 
Table 1 Proposal for the new classification (revised)

\begin{tabular}{llllll}
\hline Group/grade & $\begin{array}{l}\text { Limbal } \\
\text { involvement } \\
\text { (clock hours) }\end{array}$ & $\begin{array}{l}\text { Maximal } \\
\text { basal } \\
\text { diameter } \\
(\mathrm{mm})\end{array}$ & Imaging findings & Treatment recommended & Risk with upfront surgical \\
excision
\end{tabular}

Grade I: OSSN with no invasion into ocular coats clinically $\%$ on imaging (UBM)

\begin{tabular}{|c|c|c|c|c|c|}
\hline A (Small) & $\leq 3$ & $\leq 5$ & $\begin{array}{l}\text { No invasion into } \\
\text { ocular coats/globe } \\
\text { on UBM }\end{array}$ & Surgical excision with edge control & Minimal risk of side effects \\
\hline B (Large) & $>3$ to $<6$ & $5-15$ & $\begin{array}{l}\text { No invasion into } \\
\text { ocular coats/globe } \\
\text { on UBM }\end{array}$ & Immunotherapy/Immunoreduction $\mathrm{f} / \mathrm{b}$ surgical excision & $\begin{array}{l}\text { Moderate risk of side effects } \\
\text { (Limbal stem cell deficiency, } \\
\text { symblepharon formation) }\end{array}$ \\
\hline C (Diffuse) & $\geq 6$ & $\geq 15$ & $\begin{array}{l}\text { No invasion into } \\
\text { ocular coats/globe } \\
\text { on UBM }\end{array}$ & Immunoreduction with interferons $\mathrm{f} / \mathrm{b}$ surgical excision & $\begin{array}{l}\text { High risk of side effects, high risk } \\
\text { of recurrence }\end{array}$ \\
\hline
\end{tabular}

Grade II: OSSN with invasion into ocular coats (sclera/corneal stroma) on imaging

Any Any Scleral or corneal Excision with lamellar sclerectomy or keratectomy stromal invasion on +cryotherapy of margins and base/adjuvant plaque UBM brachytherapy for residual tumor at base

Grade III: OSSN with intraocular invasion

$\begin{array}{lll}\text { Any Any } \quad \begin{array}{l}\text { Intraocular } \\ \text { involvement present } \\ \text { (clinically/UBM) }\end{array} & \end{array}$

Grade IV: OSSN with intraorbital extension

Any Any Orbital extension on Exenteration CT/MRI

$U B M$ ultrasound biomicroscopy, $C T$ computed tomography scan, $M R I$ magnetic resonance imaging, $f / b$ followed by

${ }^{\mathrm{a} C}$ Clinically as suggested by mobility of conjunctival lesions in non-limbal tumors

no or limited invasion has changed significantly. Surgery is mainly replaced with topical therapy or is used for residual disease after chemoreduction or immunoreduction. The proposed classification helps to decide treatment especially in this category. I thank the authors for picking up the printing error in Table 1 (grade II tumors are tumors with extension into ocular coats on UBM or clinically, the same has been corrected in the revised table).

Till the time, further studies evaluate and define the role of other possible treatment options for intraocular and orbital disease like external beam radiotherapy, brachytherapy, or intraorbital interferon injections for a possible staged management approach; this classification may help guide treatment with the more established treatment options $[5,6]$.

\section{Compliance with ethical standards}

Conflict of interest The authors declare that they have no conflict of interest.

\section{References}

1. McGrath LA, Salvi SM. Comment on: Proposal for a new classification for ocular surface squamous neoplasia. Eye. 2018. https:// doi.org/10.1038/s41433-018-0240-y

2. Shields CL, Chien JL, Surakiatchanukul T, Sioufi K, Lally SE, Shields JA. Conjunctival tumors: review of clinical features, risks, biomarkers, and outcomes - The 2017 J. Donald M. Gass Lecture. Asia Pac J Ophthalmol (Phila). 2017;6:109-20.

3. Walsh-Conway N, Conway RM. Plaque brachytherapy for the management of ocular surface malignancies with corneoscleral invasion. Clin Exp Ophthalmol. 2009;37:577-83.

4. Arepalli S, Kaliki S, Shields CL, Emrich J, Komarnicky L, Shields JA. Plaque radiotherapy in the management of scleral-invasive conjunctival squamous cell carcinoma: an analysis of 15 eyes. JAMA Ophthalmol. 2014;132:691-6.

5. Meel R, Dhiman R, Sen S, Sharma S. Immunoreduction of locally advanced orbito-conjunctival squamous cell carcinoma with intraorbital interferon alpha-2b injection: a globe saving approach. Clin Exp Ophthalmol. 2018;46:87-88.

6. Murthy R, Gupta H, Krishnatry R, Laskar S. Electron beam radiotherapy for the management of recurrent extensive ocular surface squamous neoplasia with orbital extension. Indian J Ophthalmol. 2015;63:672-4. 


\title{
The positive impact of e-mail referrals on access to specialist macula services
}

\author{
Christopher King ${ }^{1} \cdot$ John Logan $\mathbb{D}^{2} \cdot$ Nicole $\operatorname{Lim}^{2} \cdot$ Colm Andrews $^{1} \cdot$ Susan M. Downes ${ }^{1,3}$
}

Received: 16 August 2018 / Accepted: 5 September 2018 / Published online: 19 October 2018

(c) The Royal College of Ophthalmologists 2018

Patients suspected of having neovascular age-related macular degeneration (nAMD) requires rapid access to specialist clinics for confirmatory diagnosis and subsequent treatment. The Royal College of Ophthalmologists (RCOphth) guidelines [1] recommend $<7$ days delay between initial referral in primary care to assessment with a specialist in macular disease, and for those patients requiring treatment $<7$ days delay between specialist consultation and initial treatment. In order to achieve this the Oxford Eye Hospital utilises the 'Wet AMD rapid access referral form' developed by the Thames Valley Macular Group, adopted by the RCOphth and endorsed by the Macular Society and RNIB for all referrals to the dedicated rapid-access clinic for patients with suspected nAMD [2]. To address the concern that rapid-access referrals were being missed and booked into routine clinics, a streamlined e-mail referral system was created using a dedicated nhs.net email account from August 2014.

Despite this system being widely shared with primary care providers. many referrals to our service are via other routes. We have audited the delay in access to specialist clinic for each referral route for 98 new AMD referrals seen in clinic between January and March 2018. Only 33.7\% of referrals were received thorough the dedicated e-mail address, with $19.4 \%$ via the walk-in eye casualty service or other sub-specialist clinics and the remaining $46.9 \%$ via the patients' general preactitioners (GPs). Figure 1 shows

Susan M. Downes

susan.downes@ouh.nhs.uk

1 Oxford Eye Hospital, John Radcliffe Hospital, Oxford University Hospitals NHS Foundation Trust, Headley Way,

Oxford OX39DU, UK

2 Oxford University Medical School, Medical Sciences Divisional Office, University of Oxford, John Radcliffe Hospital, Oxford OX39DU, UK

3 Nuffield Laboratory of Ophthalmology, Nuffield Department of Clinical Neurosciences, John Radcliffe Hospital, Headley Way, Oxford OX39DU, UK the delay between referral and first specialist review for patients in each of the three referral groups. No patients referred via their (GP) were seen within the 7 days target. The differences in the proportions of patients seen between the e-mail referral group and GP referral group within 2 weeks $(p=0.0014)$ and within 3 weeks $(p<0.0001)$ were statistically significant (chi-squared test).

Overall $28.6 \%$ of patients referred were found to have nAMD in at least one eye. Figure 2 shows the delay between referral and specialist review for those patients found to have nAMD and those with other diagnoses. It shows that many patients who are subsequently confirmed to have nAMD are facing delays in accessing specialist review.

This work shows that, while there are other challenges in seeing patients in a timely manner, the use of a dedicated email referral system for nAMD can significantly reduce delays in patients being seen in specialist macular clinics compared to traditional GP-centred referral systems, and the importance of reinforcing the use of this system by optometrists and other providers of primary ophthalmic services in the community. It also highlights the need for more training of community optometrists to ensure that the rapid access pathway is used only for nAMD.

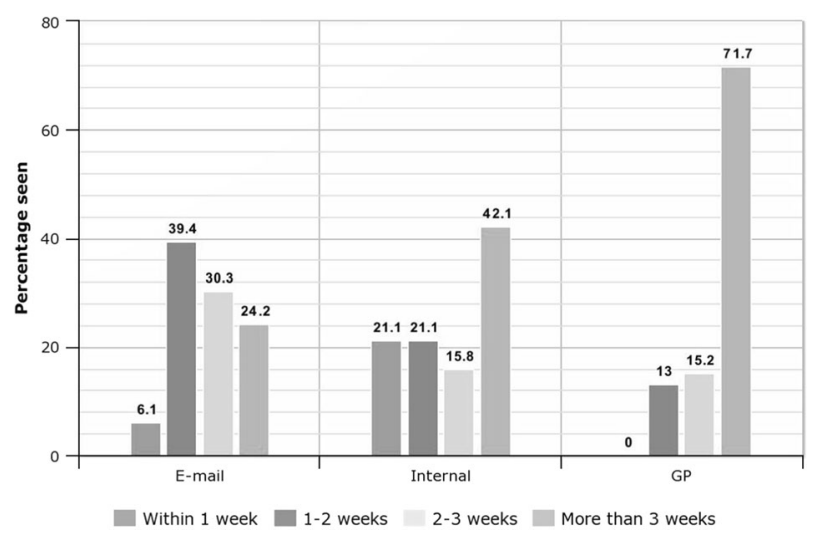

Fig. 1 Delay between referral and specialist review by referral type 


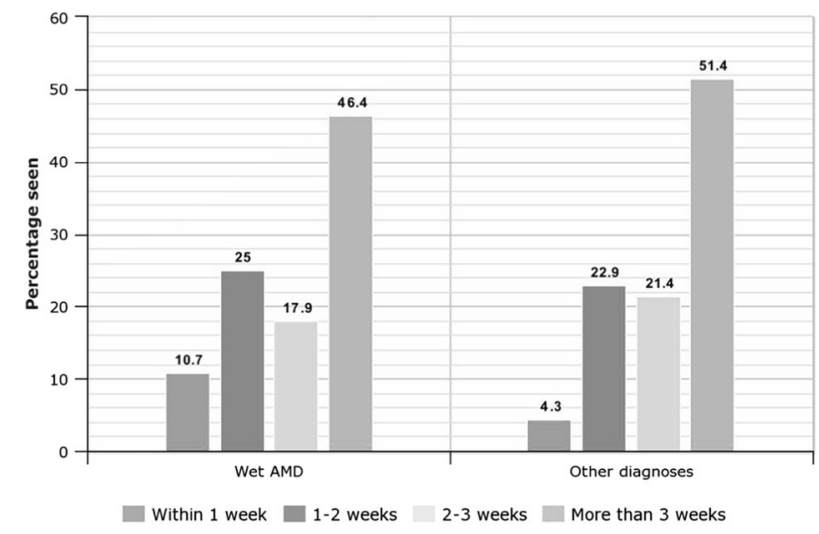

Fig. 2 Delay between referral and specialist review by diagnosis

Acknowledgements We thank the Thames Valley Macular Group Rapid Access Guidelines developed by SM Downes, C Moorman, SL Watson and L Jenkins in collaboration with the Oxfordshire LOC.

\section{Compliance with ethical standards}

Conflict of interest Susan Downes Disclosure: PI on relevant commercially sponsored trials in last 5 years: Novartis, Bayer, and
Alcon. Prof Susan Downes has received honoraria in the past 15 years from Novartis and Bayer for speaking at educational meetings, as well as travel expenses from Ely Lilly as chair for diabetic retinopathy screening meetings, and Novartis to attend educational meetings in Medical Retina pre 2010. She is a PI on a number of commercial trials (Novartis, Roche, Bayer, Allergen, among others), and has received a bursary for a research nurse practitioner, and equipment (visudyne pump for photodynamic therapy) and funding to carry out genetic testing for a research project from Novartis. She also has has been a co-PI or Pl on grants from Wellcome, UK Fighting Blindness RP, Fight for Sight and UK NIHR/CRN funding. The remaining authors declare that they have no conflict of interest.

\section{References}

1. The Royal College of Ophthalmologists. Age-Related Macular Degeneration: Guidelines for Management 2013. https://www. rcophth.ac.uk/wp-content/uploads/2014/12/2013-SCI-318RCOphth-AMD-Guidelines-Sept-2013-FINAL-2.pdf

2. The Royal College of Ophthalmologists. Wet AMD Rapid Access Referral Form 2010. https://www.rcophth.ac.uk/wp-content/uploa ds/2015/04/2010-SCI-048-AMD-Electronic-Referral-Form-edited. pdf

\title{
Accuracy of pinhole visual acuity at an urban Indian hospital
}

\author{
Rajesh S. Kumar ${ }^{1,2} \cdot$ Mahalakshmi V. Rackenchath ${ }^{1} \cdot$ A. V. Sathidevi ${ }^{1} \cdot$ Sriharsha Nagaraj $^{1} \cdot$ Caitlin A. Moe $^{3}$. \\ Robert L. Stamper ${ }^{4}$ Jeremy D. Keenan $\mathbb{1 D}^{3,4}$
}

Received: 16 August 2018 / Accepted: 7 September 2018 / Published online: 19 October 2018

(c) The Royal College of Ophthalmologists 2018

The World Health Organization-endorsed rapid assessment of avoidable blindness (RAAB) survey employs pinhole acuity to distinguish between refractive error versus conditions not correctable with eyeglasses, but few studies have validated this approach [1].

Jeremy D. Keenan

jeremy.keenan@ucsf.edu

1 Narayana Nethralaya Eye Hospital, Bangalore, India

2 Cleveland Clinic Abu Dhabi, Abu Dhabi, UAE

3 Francis I Proctor Foundation, University of California, San Francisco, CA, USA

4 Department of Ophthalmology, University of California, San Francisco, CA, USA

\section{Methods}

Ethical committees at the University of California, San Francisco and Narayana Nethralya Eye Hospital approved this study. A consecutive series of patients aged $\geq 50$ years visiting the refraction clinic at Narayana Nethralaya Eye Hospital (Bangalore, India) in September 2015 had presenting vision and pinhole vision assessed using an ETDRS chart in a fully illuminated room, and then had a manifest refraction by an experienced optometrist. Analyses are reported with bootstrapped $95 \%$ confidence intervals with resampling at the participant level to account for nonindependence of eyes. 


\section{Results}

We assessed 204 eyes from 104 individuals (mean age $63.4 \pm 7.8$ years, $51.9 \%$ female). Mean spherical equivalent after manifest refraction was -0.31 (95\% confidence interval $[\mathrm{CI}]:-0.07$ to -0.60$)$. Mean uncorrected visual acuity was $0.43 \log$ MAR units (95\% CI: 0.38-0.49), mean best corrected visual acuity (BCVA) was 0.16 (95\% CI: $0.13-$ 0.19 ) and mean pinhole acuity was 0.15 (95\% CI: 0.12 0.18). Pinhole acuity had high agreement with BCVA (intraclass correlation coefficient $0.97,95 \%$ CI: 0.96-0.98; Fig. 1). On average, pinhole acuity was less than a letter different from BCVA (mean 0.4 letters better, $95 \%$ limits of agreement by Bland-Altman method: four letters worse to five letters better). The magnitude of improvement on pinhole testing was correlated with the magnitude of spherical equivalent from refraction (Spearman's rho $=0.68, P<$ 0.001; Fig. 2).

Of 204 eyes, 21 (10.3\%) had visual impairment even after subjective refraction (BCVA worse than 20/60). When treated as a diagnostic test for visual impairment not correctable with eyeglasses, pinhole acuity provided high discriminative ability, with an area under the receiver operating characteristics (ROC) curve of 0.99 (95\% CI: 0.97-1.0). Pinhole acuity worse than $20 / 60$ was $85.7 \%$ sensitive (95\% CI: $59.1-100 \%)$ and $100 \%$ specific $(98.0-100 \%)$ for detecting visual impairment not correctable with glasses, and had a positive predictive value of $100 \%$ (95\% CI: $81.4-100 \%)$.

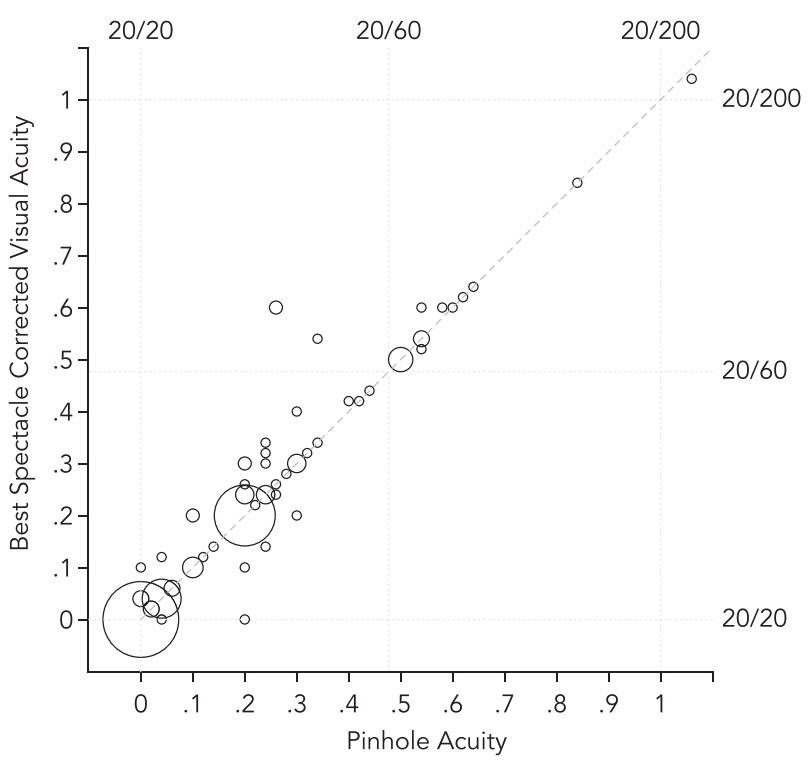

Fig. 1 Correlation between measurements of best spectacle corrected visual acuity and pinhole acuity. Points are weighted; the area of the circles represents the number of observations at each coordinate

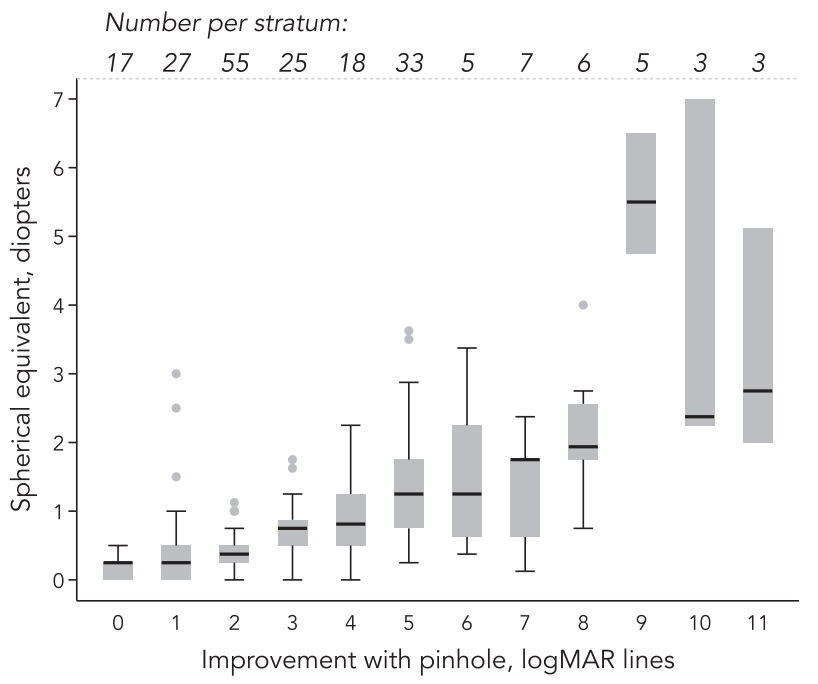

Fig. 2 Relationship between improvement with pinhole occlusion and refractive error. Eyes were stratified according to how many lines of improvement were achieved with pinhole occlusion. The distribution of spherical equivalent for each stratum is depicted as a box-andwhiskers plot

\section{Conclusions}

Previous studies of patients with diabetic retinopathy or low vision found that pinhole acuity was biased relative to BCVA, with pinhole acuity $\sim 1$ line worse than BCVA $[2,3]$. In contrast, we found essentially no bias in this population from a refraction clinic in India. The reason for the discrepancy is unclear, but the pinhole occluder may cause more visual degradation in those with retinal pathology than in a general population like ours [2]. The estimates of sensitivity and specificity of pinhole acuity as a test for visual impairment are consistent with prior reports, and suggest that estimates of refractive error based on pinhole occlusion should not overestimate the prevalence of disease [4].

In summary, pinhole acuity agreed well with BCVA and was a specific test for visual impairment not correctable with eyeglasses. These results suggest that pinhole occlusion is a valid gauge of refractive error in the RAAB survey or other community-based surveys.

Funding This work was funded by the Fortisure Foundation, That Man May See, and Research to Prevent Blindness.

\section{Compliance with ethical standards}

Conflict of interest The authors declare that they have no conflict of interest. 


\section{References}

1. Kuper H, Polack S, Limburg H. Rapid assessment of avoidable blindness. Community Eye Health. 2006;19:68-9.

2. Eagan SM, Jacobs RJ, Demers-Turco PL. Study of luminance effects on pinhole test results for visually impaired patients. Optom Vis Sci. 1999;76:50-8.
3. Sun JK, et al. Visual acuity testing using autorefraction or pinhole occluder compared with a manual protocol refraction in individuals with diabetes. Ophthalmology. 2011;118:537-42. https://doi.org/ 10.1016/j.ophtha.2010.07.022

4. Loewenstein JI, Palmberg PF, Connett JE, Wentworth DN. Effectiveness of a pinhole method for visual acuity screening. Arch Ophthalmol. 1985;103:222-3.

\title{
Time to drop the phenylephrine from the paediatric cycloplegia protocol: informing practice through audit
}

\author{
Sue Llewellyn ${ }^{1,2} \cdot$ Payal Khandelwal $^{2,3} \cdot$ Selina Glaze ${ }^{2,3} \cdot$ Peter Thomas $^{2,4,5} \cdot$ Annegret Dahlmann-Noor $\mathbb{D}^{2,4,5}$
}

Received: 25 September 2018 / Accepted: 4 October 2018 / Published online: 23 October 2018

(c) The Royal College of Ophthalmologists 2018

Cycloplegic retinoscopy is the gold standard refraction approach in children. Various protocols are in use to achieve cycloplegia during outpatient appointments. Many use not only the muscarinergic antagonist, cyclopentolate (CP), which paralyses the ciliary muscle, but also the alphaadrenergic agonist, phenylephrine (PE), which as a mydriatic paralyses the iris constrictor, but has no effect on the ciliary muscle. Whilst mydriasis facilitates visualisation of the retinoscopy reflex, there is a risk of underestimating hypermetropia. Published evidence suggests that repeated

This work was presented at the annual meeting of the British Isles Paediatric Ophthalmology and Strabismus Association, Manchester, October 3-5, 2018.

\section{Annegret Dahlmann-Noor}

annegret.dahlmann-noor@nhs.net

Bedford Hospital Trust, Kempston Road, Bedford, UK

2 Moorfields at Bedford Hospital, Children's Eye Clinic, Enhanced Services Centre, Kimbolton Road, Bedford, UK

3 Cambridgeshire Community Services NHS Trust, Unit 3, Meadow Lane, St Ives PE27 4LG, UK

4 Paediatric Service, Moorfields Eye Hospital, 162 City Road, London EC1V 2PD, UK

5 NIHR Biomedical Research Centre at Moorfields Eye Hospital and UCL Institute of Ophthalmology, 162 City Road, London EC1V 2PD, UK instillation of $\mathrm{CP}$ only is effective even for brown and very dark irides [1-3].

To develop a Patient Group Directive (PGD) we carried out a two-cycle audit (CA18/PA/02). The first round evaluated our current protocol: blue iris, CP $1 \%$ once $(0.5 \%$ if age $<3$ months); brown iris, CP/PE $2.5 \%$ once; very dark iris, CP/PE twice, $10-15$ min apart; repeated if pupils still constrict on pentorch illumination. As standard, we set full dilation in $90 \%$ within $30 \mathrm{~min}$, i.e. the level expected for blue irides with a single $\mathrm{CP}$ drop [4].

In the second round, we only included children with brown or very dark iris, administering $\mathrm{CP}$ twice or three times, respectively, $10-15$ min apart.

Table 1 Ethnic background and iris colour of children assessed in this audit

\begin{tabular}{llllll}
\hline & \multicolumn{2}{l}{ Round 1 } & & \multicolumn{2}{l}{ Round 2 } \\
\cline { 6 - 6 } & $n$ & $\%$ & & $n$ & $\%$ \\
\hline Ethnicity & & & & \\
Afro-Caribbean & 5 & 9 & 10 & 20 \\
Asian & 7 & 13 & 19 & 37 \\
Caucasian & 44 & 79 & 20 & 39 \\
Chinese & & & 1 & 2 \\
Other & & & 1 & 2 \\
Iris colour & 32 & 57 & & \\
Blue & 11 & 20 & 14 & 27 \\
Brown & 13 & 23 & 37 & 73 \\
Very dark & & & & \\
\hline
\end{tabular}




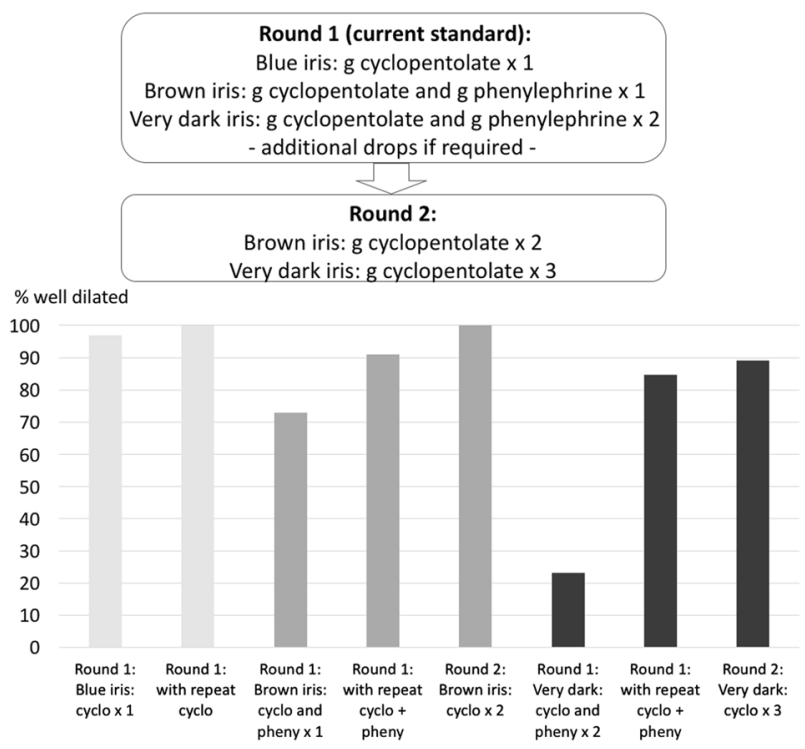

Fig. 1 Proportion of well-dilated pupils in rounds 1 and 2 of the audit. Eyes with blue irides are well dilated after one application of cyclopentolate $(\mathrm{CP})$. In brown irides, application of $\mathrm{CP}$ twice increases the proportion of well-dilated pupils, and in very dark irides, application of $\mathrm{CP}$ three times is effective. Phenylephrine does not appear to enhance this effect

We assessed pupil constriction to pentorch illumination, standard when deciding whether additional drops are required. We also noted time from instillation of the first drop to pentorch test.

We evaluated 107 consecutive children (Table 1). Round 1 found that pupils were dilated in $97 \%$ of children with blue, $73 \%$ with brown, and 23\% with very dark iris (Fig. 1), at a mean 24 min (SD 18) after first drop. Repeat instillation increased success to $100 \%, 91 \%$, and $85 \%$, respectively. In the second round, pupil dilation was achieved in all children with brown iris, and in $89 \%$ with very dark iris, at a mean 32 min (SD 13) after first instillation.

Our use of pupil constriction as outcome measure, which assesses mydriasis, not cycloplegia, is a limitation. However, as we found that better dilation is achieved without the use of PE, this audit has changed our practice and contributed positively to the development of the PGD.

Acknowledgements This work was supported by the National Institute for Health Research (NIHR) Moorfields Biomedical Research Centre. The views expressed are those of the authors and not necessarily those of the NHS, the NIHR or the Department of Health.

\section{Compliance with ethical standards}

Conflict of interest The authors declare that they have no conflict of interest.

\section{References}

1. Farhood Q. Cycloplegic refraction in children with cyclopentolate versus atropine. J Clin Exp Ophthalmol. 2012;3:239.

2. Ebri A, Kuper H, Wedner S. Cost-effectiveness of cycloplegic agents: results of a randomized controlled trial in nigerian children. Invest Ophthalmol Vis Sci. 2007;48:1025-31.

3. Celebi S, Aykan U. The comparison of cyclopentolate and atropine in patients with refractive accommodative esotropia by means of retinoscopy, autorefractometry and biometric lens thickness. Acta Ophthalmol Scand. 1999;77:426-9.

4. Zurevinsky J, Sawchuk K, Lim HJ, Lee CH, Rubab S. A clinical randomized trial comparing the cycloplegic effect of cyclopentolate drops applied to closed eyelids versus open eyelids. Am Orthopt J. 2016;66:114-21. 


\title{
Do corneas melt in the Scottish summer? A 10-year review of corneal perforations requiring cyanoacrylate gluing in theatre at a tertiary referral centre
}

\author{
Magdalena Edington $^{1} \cdot$ Julie Connolly ${ }^{1}$ David Lockington (i) $^{1}$ \\ Received: 1 October 2018 / Accepted: 8 October 2018 / Published online: 24 October 2018 \\ (c) The Royal College of Ophthalmologists 2018
}

Previous studies have identified the potential role of temperature and barometric pressure in contributing to a flare of inflammatory symptoms in susceptible rheumotological patients, but this association is controversial and not definitive $[1,2]$. Joint inflammation is due in part to the proinflammatory role of matrix metalloproteinases (MMPs); enzymes which also have a destructive role towards the corneal extracellular matrix [3]. We wanted to perform a 10-year review of corneal perforations requiring cyanoacrylate gluing in theatre at our university tertiary referral centre to assess for any seasonal association [4].

Patients who underwent emergency gluing procedures in theatre for corneal perforation at Gartnavel General Hospital, Glasgow between 2008 and 2017 were identified from theatre logbooks and their electronic records retrospectively analysed. Audit data included patient demographics, date of perforation, ocular co-morbidities and any associated systemic conditions.

There were 65 episodes in 43 patients of corneal perforation requiring gluing in this 10-year period. All identified cases were treated with application of cyanoacrylate glue. The majority of perforations $(27 / 43 ; 63 \%)$ occurred in patients $>60$ years of age, and of female gender $(25 / 43 ; 58 \%)$. Of the 43 patients, ten had an underlying systemic connective tissue disorder such as rheumatoid arthritis (RA) or systemic lupus erythematosus (SLE), nine had infectious keratitis, six had an inflammatory ocular surface (rosacea, ocular cicatrical pemphigoid, blepharitis), five were graft failures, three had corneal ectasias, seven fell into the 'other causes' category (including trauma, exposure, post-LASIK), and no clear cause was documented in three patients. There

David Lockington

davidlockington@hotmail.com

1 Tennent Institute of Ophthalmology, Gartnavel General Hospital, 1053 Great Western Road, Glasgow G12 0YN, UK were five patients who had three or more episodes of perforation; underlying diagnoses in these patients included Logic syndrome, SLE and rosacea keratitis.

There was a slightly higher incidence of corneal perforations in the spring and summer months (17 episodes in spring, 22 summer, 13 autumn, 13 winter). This association did not reach statistical significance (Chi-square $p=0.485$ ). With respect to those patients with underlying systemic connective tissue disorders, eight episodes occurred in the spring/summer months and eight in autumn/winter (see Figs. 1 and 2).

Average temperatures in Scotland range from 3 to 15 degrees Celsius $\left({ }^{\circ} \mathrm{C}\right)$, with mean and median temperatures of $9^{\circ} \mathrm{C}$. [See 'Scotland's Environment' from the Scottish Government, accessible at https://www. environment.gov.scot/media/1185/climate-climate.pdf].

We did not identify an increase in corneal perforations according to traditionally colder seasons in Scotland; rather, there was a slightly higher incidence in the spring/ summer. This was not statistically significant, possibly due to the small sample size and the lack of extreme temperatures between the Scottish seasons. We could not identify any reports linking weather conditions and corneal melting in the ophthalmic literature. Diseasemodifying anti-rheumatic drugs have previously been shown to improve the visual outcome in rheumatological patients with corneal melting, but this only implicates the presence of cellular inflammation, not the contributing factors for a flare in the underlying inflammatory condition [5]. A larger prospective study analysing these factors would be required to explore any link between seasonal variation and corneal melting leading to perforation.

Acknowledgements A version of this study was an oral presentation at the Scottish Ophthalmological Club, Stirling, Scotland in September 2017. 
Fig. 1 Graph illustrating number of corneal perforations requiring gluing by month of year from 2008-2017

Fig. 2 Clinical photographs illustrating use of cyanoacrylate glue in the management of corneal perforations, either as direct application or in association with a plastic disc (punched from the plastic drape by a skin biopsy trephine)
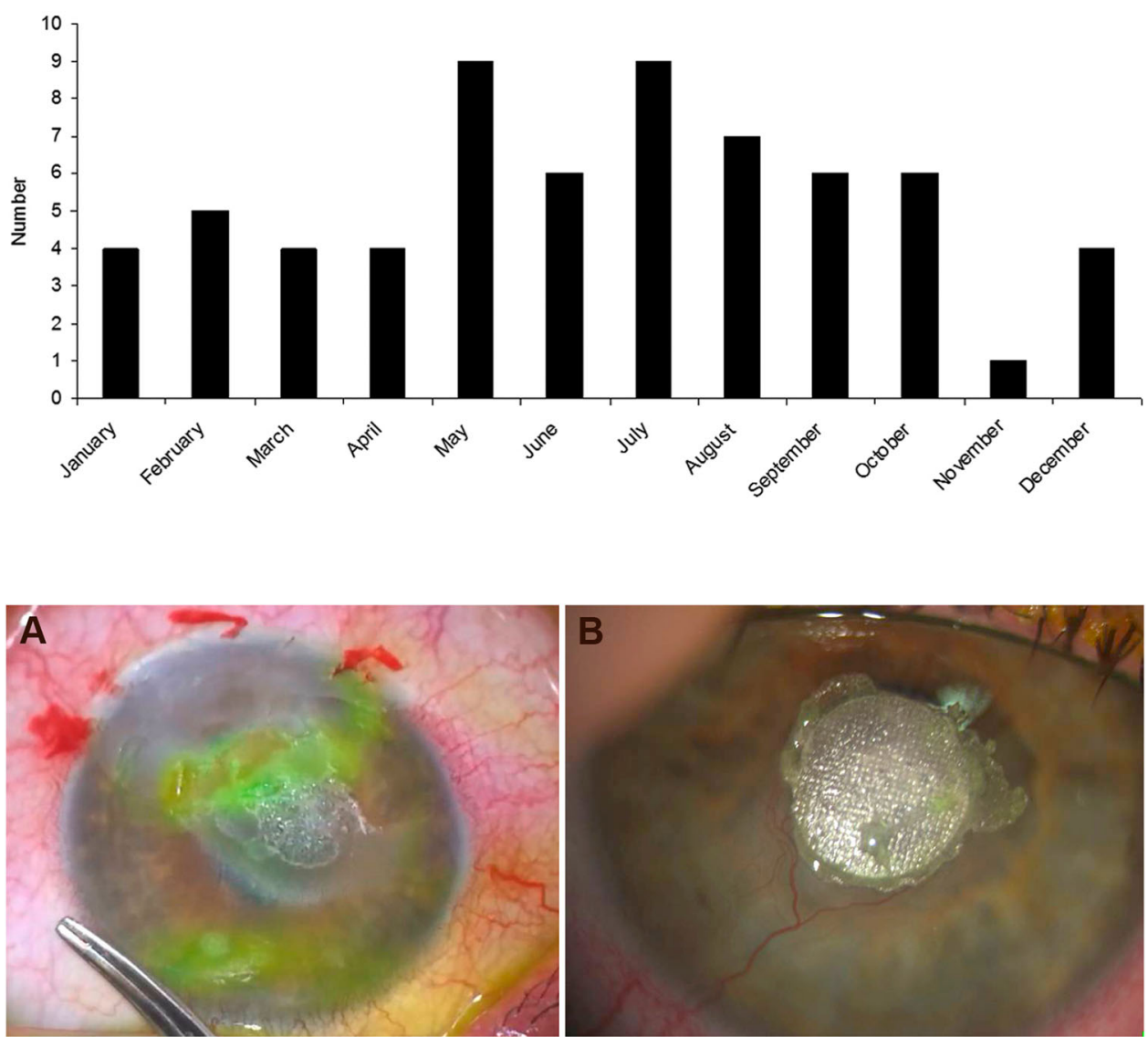

\section{Compliance with ethical standards}

Conflict of interest The authors declare that they have no conflict of interests.

\section{References}

1. Patberg WR, Rasker JJ. Weather effects in rheumatoid arthritis: from controversy to consensus. A review. J Rheumatol. 2004;31:1327-34.
2. Savage EM, McCormick D, McDonald S, Moore O, Stevenson M, Cairns AP. Does rheumatoid arthritis disease activity correlate with weather conditions? Rheumatol Int. 2015;35: 887-90.

3. Sakimoto T, Sawa M. Metalloproteinases in corneal diseases: degradation and processing. Cornea. 2012;31 (Suppl 1): S50-6.

4. Jhanji V, Young AL, Mehta JS, Sharma N, Agarwal T, Vajpayee RB. Management of corneal perforation. Surv Ophthalmol. 2011;56:522-38.

5. Saripalli L, Harrington TM, Notz RG, Torretti D. Corneal melt in rheumatic disorders: effect of disease-modifying antirheumatic drugs on morbidity. J Clin Rheumatol. 2005;11:134-9. 


\title{
latrogenic cannula-associated ocular injuries during anterior segment surgery: time to re-think luer-lock design?
}

\author{
Craig Wilde $^{1} \cdot$ Andrew R. Ross $^{1} \cdot$ Gavin Orr ${ }^{1} \cdot$ Harminder Dua $\mathbb{D}^{1}$ \\ Received: 21 August 2018 / Accepted: 7 September 2018 / Published online: 25 October 2018 \\ (c) The Royal College of Ophthalmologists 2018
}

Cataract surgeons are aware of potential iatrogenic ocular injuries from accidental cannula release during injections of solutions or ophthalmic viscosurgical device (OVD) [1, 2]. Prevention advice is simple. Surgeons and theatre nurse practitioners should be aware of the complication, taught to check instrument security and comply with manufacturers user instructions. Surgeons' should firmly hold the cannula base. Universal adoption of luer-lock syringes has been established. Despite these measures however, events still occur.

Although rare (with possible under reporting), incidence during cataract surgery is between 0.009 [1] and $0.07 \%$ [2]. Serious ocular injuries can occur with visual loss being reported particularly with retinal damage [2]. Despite efforts to increase awareness, events still occur. It appears the standard advice to check for tight adherence and hold the cannula hub during injection is not enough to adequately mitigate risk. Even apparently tight fitting cannulas have been reported to dislodge [3], with reports of both luer-lock adapter and cannula becoming detached from the syringe, acting as a projectile [4]. It is still possible that surgeon and theatre nurse both fail to screw and tighten the luer-lock connection or cross threading could occur.

The author $(\mathrm{CW})$ has first-hand experience of being handed an OVD with the luer-lock being accidentally removed by the theatre nurse. Instead of the 'tip cap' of an Amvisc (Bausch and Lomb, Kingston Upon Thames, UK) being screwed off, the entire luer-lock system was removed (by a pulling motion) and cannula affixed, contrary to the manufacturer's instructions (Fig. 1). A twisting/tightening motion can be performed by the surgeon, but unless direct visual inspection of the device is performed, it will not be immediately evident that the luer-lock has been removed. This poses a potential risk and cataract surgeons must be aware that inadvertent detachment of luer-locks is possible (depending on the individual product). It is the authors' opinion that device design should change making inadvertent removal of luer-locks impossible. If iatrogenic cannula injuries are to be completely eradicated from practice, closer liaison with instrument manufacturers is required. Development of integrated syringe-cannula systems to eliminate all possibilities of inadequately tightening or inappropriately removing luer-locks is needed. Simple statements that luer-locks never fail will not avoid future harm if human performance shortfalls cannot be eliminated from every possible step. The safest way to do this is at the manufacturing stage by moulding one-piece cannula-syringe devices, where syringe and cannula hub are moulded together eliminating the possibility of detachment by force or inadequate tightening.

Acknowledgements Photographs taken by Emily Hogan, Queen's Medical Center, Nottingham.

\section{Compliance with ethical standards}

Conflict of interest The authors declare that they have no conflict of interest.
Harminder Dua

harminder.dua@nottingham.ac.uk

1 Ophthalmology and Visual Sciences, Division of Clinical Neurosciences, B Floor, EENT Centre, Queen's Medical Centre, University of Nottingham, Nottingham, UK 

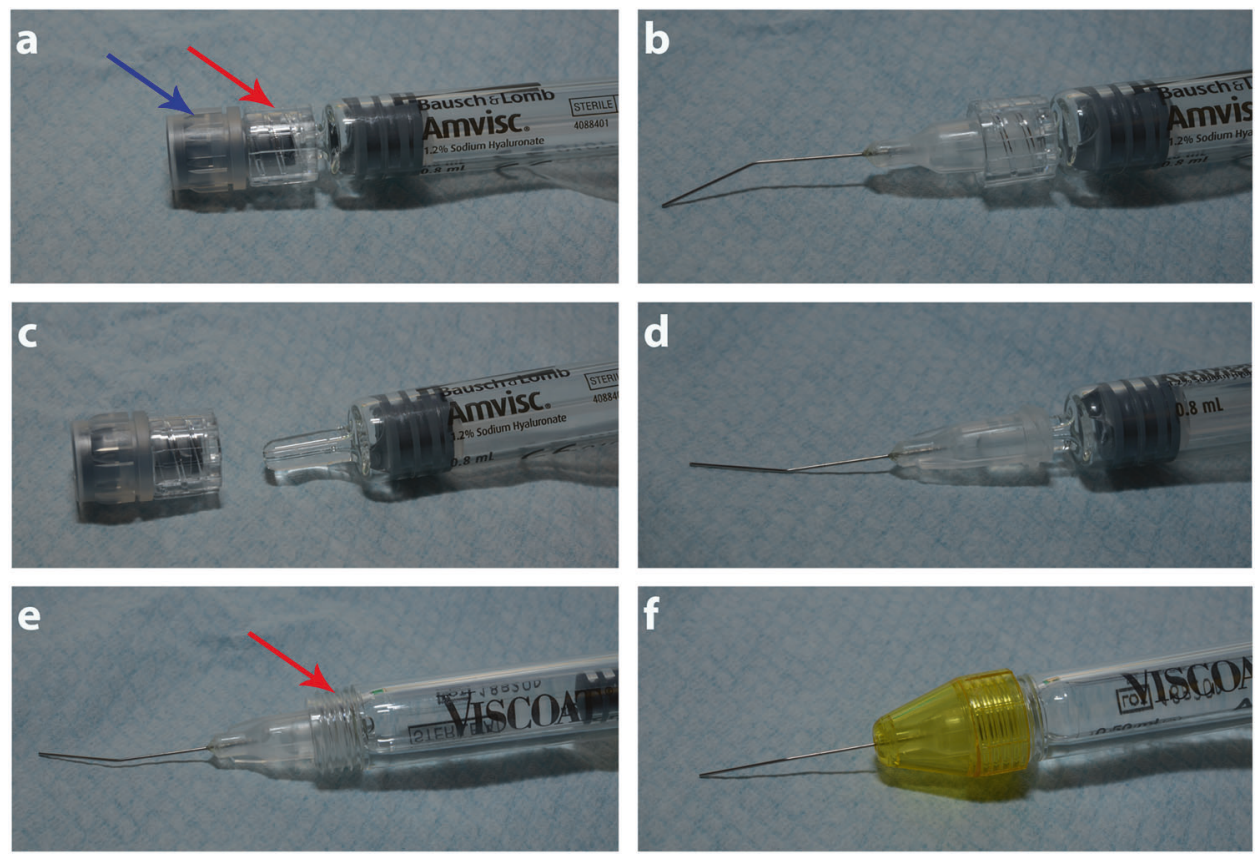

Fig. 1 Amvisc syringe assembly as provided out of packaging (a). The grey 'tip cap' (blue arrow) has to be screwed off the 'luer-lock' (red arrow) and then cannula (with luer) tightened within the luer-lock for correct assembly (b). It is possible to detach the luer-lock from the end of the Amvisc syringe, obviously going against the manufacturers instructions (c). However, this makes it possible for a surgeon to be handed an inappropriately assembled OVD that poses a danger of

forceful detachment (d). An Alcon Viscoat syringe assembly (e). The syringe tip has a non-detachable moulded luer-lock (red arrow) that cannot be removed. To act as a safeguard from failure to correctly tighten the lure-lock by rotating the cannula to the full length of the sleeve threads, the design is improved by the inclusion of a yellow cannula locking cap (f). By applying the locking cap against the cannula hub, the cannula cannot act as a projectile

\section{References}

1. Ting DS, Vaideanu-Collins D, Ellerton C. Cannula-associated ocular injuries during cataract surgery: the North East England Study. Eye. 2016;30:764-5.

2. Rumelt S, Kassif Y, Koropov M, Landa E, Marzuk F, Segal ZI, et al. The spectrum of iatrogenic intraocular injuries caused by inadvertent cannula release during anterior segment surgery. Arch Ophthalmol. 2007;125:889-92.

3. Dinakaran S, Kayarkar VV. Intraoperative ocular damage caused by a cannula. J Cataract Refract Surg. 1999;25:720-1.

4. Verbrugghe $\mathrm{A}$, Hesta $\mathrm{M}$, Gommeren $\mathrm{K}$, Daminet $\mathrm{S}$, Wuyts B, Buyse J, et al. Oligofructose and inulin modulate glucose and amino acid metabolism through propionate production in normal-weight and obese cats. Br J Nutr. 2009; 102:694-702. 